\title{
Synthesis of N-Substituted Benzamide Derivatives and their Evaluation as Antitumor Agents
}

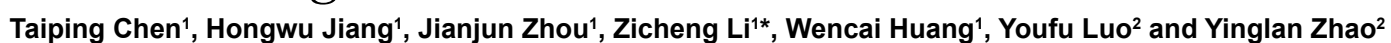

${ }^{1}$ School of Chemical Engineering, Sichuan University, Chengdu, Sichuan, China

${ }^{2}$ State Key Laboratory of Biotherapy, West China Hospital, West China Medical School, Chengdu, Sichuan, China

\begin{abstract}
A series of $\mathrm{N}$-substituted benzamide derivatives designed based on Entinostat (MS-275) were synthesized, and characterized by IR, MS, ${ }^{1} \mathrm{H}$ NMR and ${ }^{13} \mathrm{C}$ NMR. Their anti-proliferative activity in vitro against four cell lines including MCF-7, MDA-MB-231, K562 and A549 were also evaluated by the MTT assay, the results showed that several compounds displayed similar inhibitory activity compared with MS-275. Finally, binding affinity of the synthesized compounds towards targets (histone deacetylases, HDACs) was studied using molecular docking simulations, compounds $13 \mathrm{~h}$ and $13 \mathrm{k}$ were observed hydrogen bond, Van der Waals bond and hydrophobic interactions with HDAC2 and HDAC8.
\end{abstract}

Keywords: N-substituted benzamide derivative; Anti-proliferative activity; Molecular docking study

\section{Introduction}

Cancer is traditionally seen as a genetic disease; its expansion is revealed to be associated with mutations resulting in activation of oncogenes or inactivation of tumor suppressor genes [1-3]. With the deep research on gene function, however, the genetic alterations and abnormalities, epigenetic changes which are defined as heritable changes in gene function that do not involve changes in the DNA sequence, are now widely implicated in tumor onset and progression [4]. As for epigenetic regulation mechanism, it mainly contains DNA methylation, post-translational modifications of histone protein and remodeling of nucleosomes [5]. While histone deacetylases (HDACs) play a significant role in epigenetic regulation of gene expression by removing acetyl groups from $\varepsilon$-amino groups of lysine residues in the N-terminal extension of the core histones and controlling other cellular functions, such as inducing cell-cycle arrest, promoting differentiation and stimulating tumor cell death [6,7]. What's more, histone deacetylases inhibitors (HDACIs) with different structures have been developed for cancer therapy, for instance, Chidamide has been approved by FDA to cure peripheral T-cell lymphoma (PTCL), Entinostat (MS-275) is now in the clinical stage III to treat breast cancer, and so on.

Biological efficacy of HDACIs is mainly attributed to the active structure which can coordinate with the zinc ion that exists in histone protein through hydrogen bond [8,9]. Most benzamide derivatives as HDACIs for cancer treatment mainly consist of three parts: zinc binding groups (ZBG), cap and a linker [10]. Based on the strategy of structural modification of drugs, each part mentioned above could be modified to change their biological activity. For instance, Mocetinostat (MGCD0103, shown in Figure 1) which is undergoing clinical phase II for the treatment of lymphoma and bladder cancer with lower toxicity could be seen as a linker modification of MS-275 [11].

Based on modification of the zinc binding group part of MS275 , fourteen $\mathrm{N}$-substituted benzamide derivatives were synthesized, their anti-proliferative activity against MCF-7, MDA-MB-231, K562 and A549 in vitro were evaluated, and preliminary structure-activity relationship was also discussed. Molecular docking study was further employed to explain the binding affinity of the synthesized compounds towards targets. The docking data are in agreement with those bioactivity test results.

\section{Experimental}

\section{Chemistry}

All the reagents were purchased from commercial suppliers without further purification unless otherwise specified. Melting points were determined with a capillary method and are uncorrected. IR spectra were recorded on a Spectrum Two Li10014 spectrometer. ${ }^{1} \mathrm{H}$ NMR and ${ }^{13} \mathrm{C}$ NMR spectra were recorded on a Bruker Avance 400 $\mathrm{MHz}$ spectrometer. NMR spectra were recorded in DMSO-d6 or $\mathrm{CDCl}_{3}$ solutions at room temperature $\left(20 \pm 2{ }^{\circ} \mathrm{C}\right) .{ }^{1} \mathrm{H}$ and ${ }^{13} \mathrm{C}$ chemical shifts are quoted in parts per million downfield from TMS. ESI-MS spectra were recorded on a Bruker Esquire 3000 instrument. Highresolution mass spectra (HRMS) were obtained on a MicrOTOF-Q II mass spectrometer with an ESI source (Waters, Manchester). All reactions were monitored by thin-layer chromatography (TLC) and using ultraviolet light $(254 \mathrm{~nm})$. As for known compounds, only melting point and ${ }^{1} \mathrm{H}$ NMR spectra were confirmed with previously reported literatures and the main intermediates were characterized by IR, ${ }^{1} \mathrm{H}$ NMR, ${ }^{13} \mathrm{C}$ NMR spectra and mass spectra.

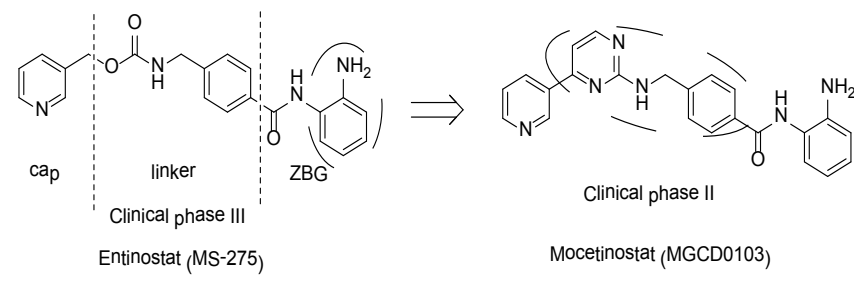

Figure 1: The structure of pre-clinical compounds MS-275 and MGCD0103.

*Corresponding author: Zicheng Li, School of Chemical Engineering, Sichuan University, Chengdu, Sichuan, China, Tel: +862885405221; E-mail: sculzc@scu.edu.cn

Received October 06, 2018; Accepted October 25, 2018; Published November 01 2018

Citation: Chen T, Jiang H, Zhou J, Li Z, Huang W, et al. (2018) Synthesis of $\mathrm{N}$-Substituted Benzamide Derivatives and their Evaluation as Antitumor Agents. Med Chem (Los Angeles) 8: 273-280. doi: 10.4172/2161-0444.1000524

Copyright: $\odot 2018$ Chen T, et al. This is an open-access article distributed under the terms of the Creative Commons Attribution License, which permits unrestricted use, distribution, and reproduction in any medium, provided the original author and source are credited. 
Scheme 1

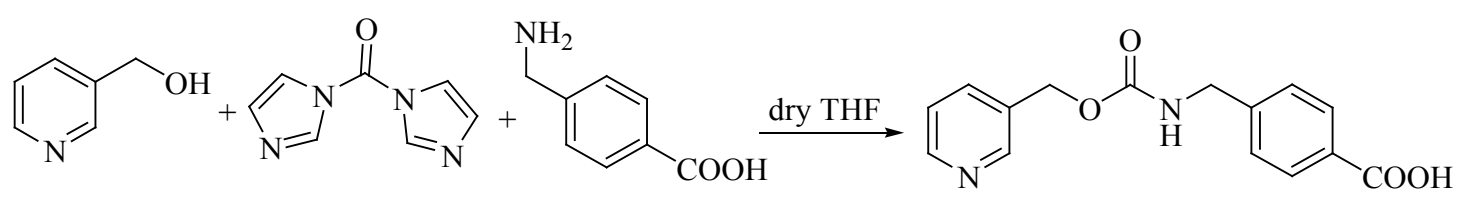

1

Scheme 2<smiles>[R]C(=O)c1ccc(N)cc1</smiles>

$\mathbf{a}: \mathrm{R}^{=} \mathrm{NH}_{2} \quad \mathbf{b}: \mathrm{R}^{=}$

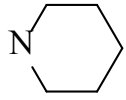

c: $\mathrm{R}=\sqrt{\mathrm{O}}$

$\mathrm{d}: \mathrm{R}=\mathrm{N}$

\section{Scheme 3}

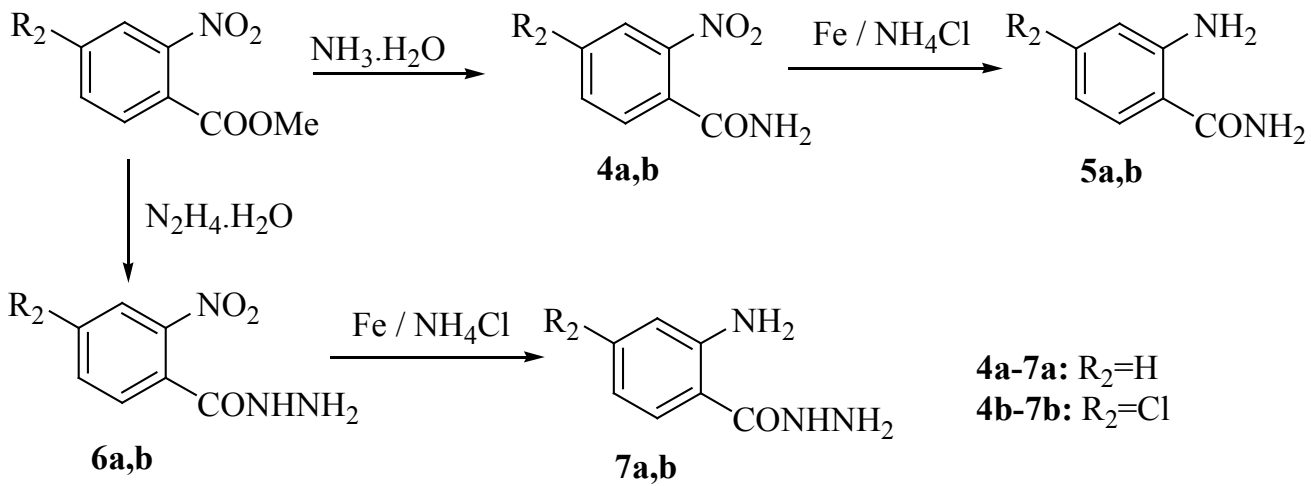<smiles>[R]C(=O)c1cc([R])ccc1O</smiles>

$\mathbf{8 a}, \mathbf{b}$ 8a,9a: $\mathrm{R}_{3}=\mathrm{H}$

8b,9b: $\mathrm{R}_{3}=\mathrm{Cl}$<smiles>[R3]c1ccc(O)c(C(=O)NN)c1</smiles>

9a,b<smiles>COC(=O)c1[X]:[Y]ccc1</smiles>

10a: $\mathrm{X}=\mathrm{C}, \mathrm{Y}=\mathrm{N}$

10b: $X=N, Y=C$ 
Scheme 4<smiles>[R]c1ccc([N+](=O)[O-])c(F)c1</smiles>

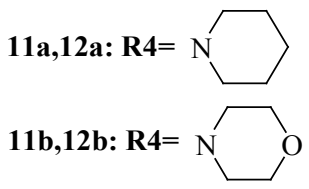

\section{Scheme 5}

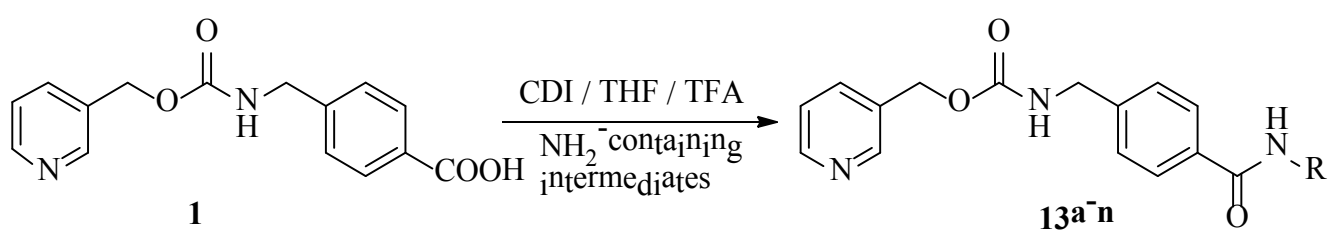<smiles>[X]c1cccc(C(=O)NC)c1</smiles>

13a: $\mathrm{X}=\mathrm{NH}_{2}$ $13 \mathrm{~b}: \mathrm{X}^{-} \mathbf{1}^{-}$piperidinyl 13c: $X={ }^{-}{ }^{-m o r p h o l i n} n_{y l}$ 13d: $X{ }^{=} 4^{-}$methyl ${ }^{-1}$ piperazinyl<smiles>CC(=O)c1ccccc1N</smiles><smiles>[X]c1cc([X])c(C(=O)NC)cc1[X]</smiles>
13f: $X{ }^{=} \mathrm{NH}_{2}, Y={ }^{=}{ }^{=} \mathrm{H}$ $13^{-} \mathrm{n}$ 13g: $X=\mathrm{NH}_{2}, Y={ }_{\mathrm{H}}, Z=\mathrm{Cl}$ 13h: $X=\mathrm{OH}^{\prime} Y={ }^{\prime}=\mathrm{H}$

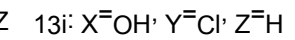

THF distilled off by adding sodium and acetophenone as indicator under $\mathrm{N}_{2}$ atmosphere and purified from water.

Synthesis of 4-[N-(pyridine-3-methoxycarbonyl) amino methyl] benzoic acid: (1)-To a suspension of $\mathrm{N}, \mathrm{N}^{\prime}$-carbonyldiimidazole (CDI, $10 \mathrm{mmol})$ in dry THF $(10 \mathrm{~mL})$ was added pyridin-3-ylmethanol $(10$ $\mathrm{mmol})$ dissolved in THF $(10 \mathrm{~mL})$ by three portions within $10 \mathrm{~min}$ at $0-10^{\circ} \mathrm{C}$, the mixture was stirred at that temperature for $1 \mathrm{~h}$. The resulting solution was added into a solution of 4-(aminomethyl)benzoic acid $(10 \mathrm{mmol})$ dissolved in sodium hydroxide solution $(10 \mathrm{~mL}, 1 \mathrm{~mol} / \mathrm{L})$ at $0-10^{\circ} \mathrm{C}$, after stirring for $5 \mathrm{~h}$ at room temperature, the solvent was evaporated and then dissolved in water $(25 \mathrm{~mL})$. The solution was acidified with $\mathrm{HCl}(5 \mathrm{~mol} / \mathrm{L})$ to adjust $\mathrm{pH} 5$ to precipitate white solid which was collected by filtration, washed with water twice and dried to give pure 1 . Yield: $83 \%$; mp: $206-208^{\circ} \mathrm{C}[14]$, $\mathrm{mp}: 207-208^{\circ} \mathrm{C}$ ).

General procedure for the preparation of 4-nitrobenzamide derivatives: (2a-d)-To a mixture of 4 -nitrobenzoic acid $(10 \mathrm{mmol})$ in thionyl chloride $(10 \mathrm{~mL})$ was added three drops of DMF, the mixture was refluxed for $1.5 \mathrm{~h}$. Then thionyl chloride was evaporated under vacuum, and $\mathrm{CH}_{2} \mathrm{Cl}_{2}(20 \mathrm{~mL})$ was added into the residual to be used next. To a mixture of amine $(12 \mathrm{mmol})$ and triethylamine $(30 \mathrm{mmol})$ in $\mathrm{CH}_{2} \mathrm{Cl}_{2}(50 \mathrm{~mL})$ was dropped slowly the acyl chloride solution prepared above in ice-water bath. The mixture was then stirred for $30 \mathrm{~min}$ at room temperature, the organic layer was separated, washed twice with salt water, and dried with anhydrous sodium sulfate, and solvent was concentrated to give the crude product, which was recrystallized from ethanol to give 2 .

4-Nitrobenzamide (2a): Creamy-white solid; yield: 77\%; mp: 198$200^{\circ} \mathrm{C}$ ([15], mp: $\left.200-202^{\circ} \mathrm{C}\right)$.

(4-Nitrophenyl)(piperidin-1-yl)methanone (2b): Yield: 83\%; mp: $119-121^{\circ} \mathrm{C}\left([16], \mathrm{mp}: 121-122^{\circ} \mathrm{C}\right)$.
Morpholino(4-nitrophenyl)methanone (2c): Yield: 81\%; mp: 101$102^{\circ} \mathrm{C}$ ([17], mp: $\left.101-102^{\circ} \mathrm{C}\right)$.

(4-Methylpiperazin-1-yl)(4-nitrophenyl)methanone (2d): Yield: 78\%; mp: $98-100^{\circ} \mathrm{C}\left([18], \mathrm{mp}: 98-100^{\circ} \mathrm{C}\right)$.

General procedure for the preparation of 4 -aminobenzamide derivatives: (3a-d)-Compound $2(10 \mathrm{mmol})$ in ethanol $(50 \mathrm{~mL})$ was hydrogenated at $0.2-0.4 \mathrm{MPa}$ catalyzed by $10 \% \mathrm{Pd}-\mathrm{C}$ for $5 \mathrm{~h}$ and recrystallized from ethanol to obtain compound 3 .

4-Aminobenzamide (3a): Brown solid; yield: $73 \%$; $\mathrm{mp}: 178-180^{\circ} \mathrm{C}$ ([19], mp: $\left.177-179^{\circ} \mathrm{C}\right)$.

(4-Aminophenyl)(piperidin-1-yl)methanone (3b): Yield 75\%; mp: 158-160 ([20], mp:158-160 $\mathrm{C})$.

(4-Aminophenyl)(morpholino)methanone (3c): Yield: 76\%; mp: $132-134^{\circ} \mathrm{C}\left([21], \mathrm{mp}: 136-138^{\circ} \mathrm{C}\right)$.

(4-Aminophenyl)(4-methylpiperazin-1-yl) methanone (3d): Yield: 70\%; mp: $144-146^{\circ} \mathrm{C}\left([22]\right.$, $\left.\mathrm{mp}: 140^{\circ} \mathrm{C}\right)$.

General procedure for the preparation of different kinds of substituted aromatic amides: (5a-b, 9a-b) and aromatic hydrazides (7a-b, 10a-b, 12a-b)-To a solution of methyl 2-nitrobenzoate, methyl salicylate, methyl 5-chlorosalicylate, or methyl 4-chloro-2nitrobenzoate $(10 \mathrm{mmol})$ in methanol $(25 \mathrm{~mL})$ was added ammonia $(15 \mathrm{~mL})$ or hydrazine hydrate $(30 \mathrm{mmol})$, the solution was stirred at $50^{\circ} \mathrm{C}$ for $48 \mathrm{~h}$, the resulting precipitate was filtered to obtain the corresponding aromatic amides or aromatic hydrazides.

The other amides or form hydrazide were similarly prepared from corresponding methyl ester.

2-Nitrobenzamide (4a): Yield: $77 \%$; mp: $173-175^{\circ} \mathrm{C}$ ([23], mp: $175-$ $\left.176^{\circ} \mathrm{C}\right)$. 
Citation: Chen T, Jiang H, Zhou J, Li Z, Huang W, et al. (2018) Synthesis of N-Substituted Benzamide Derivatives and their Evaluation as Antitumor Agents. Med Chem (Los Angeles) 8: 273-280. doi: 10.4172/2161-0444.1000524

4-Chloro-2-nitrobenzamide (4b): Yield: $80 \%$; $\mathrm{mp}: 144-146^{\circ} \mathrm{C}([24]$, $\left.\mathrm{mp}: 145-149^{\circ} \mathrm{C}\right)$.

2-Nitrobenzohydrazide (6a): Yield: $78 \%$; $\mathrm{mp}: 121-123^{\circ} \mathrm{C}([25], \mathrm{mp}:$ $\left.121-123^{\circ} \mathrm{C}\right)$.

4-Chloro-2-nitrobenzohydrazide (6b): Yield: $83 \%$; mp: $178-180^{\circ} \mathrm{C}$.

2-Hydroxybenzamide (8a): Yield: $70 \%$; mp: $140-142^{\circ} \mathrm{C}$ ([26], mp: $\left.140-142^{\circ} \mathrm{C}\right)$.

5-Chloro-2-hydroxybenzamide (8b): Yield: $79 \%$; mp: $226-227^{\circ} \mathrm{C}$ ([27], mp: $226-227^{\circ} \mathrm{C}$ ).

2-Hydroxybenzohydrazide (9a): Yield: $84 \%$; mp: $148-150^{\circ} \mathrm{C}$ ([28], $\left.\mathrm{mp}: 149-150^{\circ} \mathrm{C}\right)$.

5-Chloro-2-hydroxybenzohydrazide (9b): Yield: 78\%; mp: 211$213^{\circ} \mathrm{C}\left([29]\right.$, $\left.\mathrm{mp}: 208-210^{\circ} \mathrm{C}\right)$.

Picolinohydrazide (10a): Yield: $83 \%$; mp: $100-102^{\circ} \mathrm{C}$ ([30], mp: $\left.100.2-101.0^{\circ} \mathrm{C}\right)$.

Nicotinohydrazide (10b): Yield: $78 \%$; mp: $160-162^{\circ} \mathrm{C}$ ([30], mp: $\left.161.6-162.3^{\circ} \mathrm{C}\right)$

General procedure for the preparation of 4-substituted-2aminobenzamide and 4-substituted-2-aminobenzohydrazide: To a solution of $\mathrm{NH}_{4} \mathrm{Cl}(15 \mathrm{mmol})$ dissolved in water $(20 \mathrm{~mL})$ was added iron powder $(15 \mathrm{mmol})$, then compound 4 or 6 dissolved in ethanol ( 20 $\mathrm{mL}$ ) was added slowly into the solution with agitating, the mixture was heated to $80^{\circ} \mathrm{C}$ and stirred for $1.5 \mathrm{~h}$. The resulting mixture was filtered with diatomite and the residue was washed with ethanol $(2 \times 15 \mathrm{~mL})$, the filtrate was evaporated under vacuum to remove ethanol and the residue was extracted with dichloromethane $(2 \times 20 \mathrm{~mL})$, the combined organic layers were washed with brine once and dried with anhydrous $\mathrm{Na}_{2} \mathrm{SO}_{4}$, then evaporated to give the title compound.

2-Aminobenzamide (5a): White solid; yield: $81 \%$; mp: $110-112^{\circ} \mathrm{C}$ ([31], mp: $\left.110-112^{\circ} \mathrm{C}\right)$.

2-Amino-4-chlorobenzamide (5b): Yield: 77\%; $\mathrm{mp}$ : 180 $182^{\circ} \mathrm{C}\left([32], \mathrm{mp}: 180-182^{\circ} \mathrm{C}\right)$.

2-Aminobenzohydrazide (7a): Yield: $87 \%$; mp: $119-121^{\circ} \mathrm{C}([33]$, $\left.\mathrm{mp}: 119-121^{\circ} \mathrm{C}\right)$.

2-Amino-4-chlorobenzohydrazide (7b): Yield: 84\%; mp: 122$123^{\circ} \mathrm{C}\left([34]\right.$, mp: $\left.122-123^{\circ} \mathrm{C}\right)$.

General procedure for the preparation of 4-substituted-2fluoronitrobenzene: To a solution of 2,4-difluoronitrobenzene (35 $\mathrm{mmol})$ dissolved in ethyl acetate $(50 \mathrm{~mL})$ was added triethylamine $(40$ $\mathrm{mmol})$ and cyclic amine $(40 \mathrm{mmol})$, the solution was stirred at room temperature for $10 \mathrm{~h}$. The resulting mixture was filtered to remove the formed salt, and filtrate was washed with water twice, the organic layer was separated and dried with anhydrous $\mathrm{Na}_{2} \mathrm{SO}_{4}$, then evaporated to give the title compound.

1-(3-Fluoro-4-nitrophenyl) piperidine (11a): Yield: 70\%; mp: 127$129^{\circ} \mathrm{C}\left([35], \mathrm{mp}: 128-129^{\circ} \mathrm{C}\right)$.

4-(3-Fluoro-4-nitrophenyl) morpholine (11b): Yield: 68\%; mp: $146-148^{\circ} \mathrm{C}\left([36], \mathrm{mp}: 146-148^{\circ} \mathrm{C}\right)$.

General procedure synthesis of 4-substituted-2fluoronitroaniline: The reduction reaction was conducted according to the procedure of compound 5 .

2-Fluoro-4-(piperidin-1-yl) aniline (12a): Light brown solid; yield:

\begin{tabular}{|c|c|c|c|c|}
\hline \multirow{2}{*}{$\begin{array}{c}\text { Compound } \\
\text { No. }\end{array}$} & \multicolumn{4}{|c|}{$I C_{50}{ }^{a, b}(\mu \mathrm{M})$} \\
\hline & MCF-7 & A549 & K562 & MDA-MB-231 \\
\hline $13 a$ & $>80$ & $>80$ & $>80$ & 37.43 \\
\hline 13b & 62.17 & $>80$ & 65.31 & 71.19 \\
\hline $13 c$ & 73.46 & $>80$ & 66.29 & $>80$ \\
\hline $13 d$ & $>80$ & 18.37 & $>80$ & $>80$ \\
\hline $13 \mathrm{e}$ & $>80$ & 33.65 & 22.52 & $>80$ \\
\hline $13 f$ & $>80$ & 22.58 & 28.11 & 51.73 \\
\hline $13 \mathrm{~g}$ & $>80$ & $>80$ & $>80$ & $>80$ \\
\hline $13 \mathrm{~h}$ & 19.45 & 16.91 & 25.38 & 32.88 \\
\hline $13 i$ & 23.19 & 55.39 & $>80$ & 35.75 \\
\hline 13j & $>80$ & $>80$ & 45.35 & $>80$ \\
\hline $13 k$ & 28.73 & 36.45 & 27.66 & $>80$ \\
\hline 131 & $>80$ & $>80$ & 21.55 & 40.25 \\
\hline $13 m$ & $>80$ & $>80$ & 19.83 & 63.57 \\
\hline $13 n$ & $>80$ & $>80$ & $>80$ & $>80$ \\
\hline $\mathrm{MS}^{\mathrm{c}}$ & 17.36 & 38.79 & 18.34 & 21.31 \\
\hline
\end{tabular}

aThe $\mathrm{IC}_{50}$ values represent the concentration that causes $50 \%$ growth inhibition; ${ }^{\mathrm{b}} \mathrm{The} \mathrm{IC}_{50}$ values were the mean values of three repeated experiments, with a deviation within $20 \%$; 'MS is Entinostat (MS-275).

Table 1: $\mathrm{IC}_{50}$ value of target compounds 13a-n against tumor cells in vitro.

\begin{tabular}{|c|c|c|c|c|c|}
\hline $\begin{array}{c}\text { HDAC PDB } \\
\text { code }^{\mathbf{a}}\end{array}$ & \multicolumn{5}{|c|}{ Fitness } \\
\cline { 2 - 6 } & $\mathbf{1 3 e}$ & $\mathbf{1 3 f}$ & 13h & 13k & MS-275 \\
\hline 3max & 73.07 & 62.14 & 64.11 & 62.18 & 66.29 \\
\hline 1t69 & 59.09 & 51.06 & 52.70 & 61.32 & 57.13 \\
\hline 4bkx & 50.56 & 54.26 & 51.06 & 54.95 & 56.01 \\
\hline
\end{tabular}

aHDAC PDB code: 3max refers to HDAC2, 1 t69 refers to HDAC 8 and $4 \mathrm{bkx}$ refers to HDAC1

Table 2: Calculated binding affinity from molecular docking.
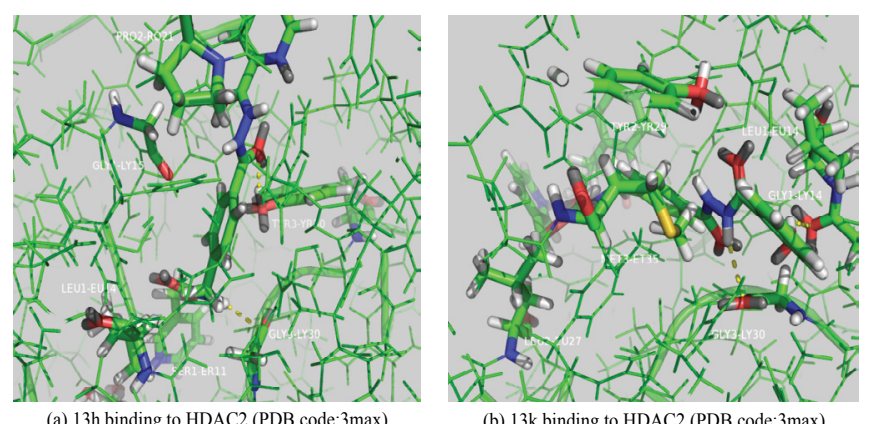

Figure 2: The docking modes of $13 \mathrm{~h}$ and $13 \mathrm{k}$ to HDAC2 respectively

72\%; mp: $85-87^{\circ} \mathrm{C}$.

2-Fluoro-4-(tetrahydro-2H-pyran-4-yl) aniline (12b): Brown solid; yield: $68 \%$; mp: $94-96^{\circ} \mathrm{C}$ ([37], $\left.\mathrm{mp}, 95-97\right)$.

Preparation of $\mathrm{N}$-(2-aminophenyl)-4-[N-(pyridin-3-yl) methoxycarbonylaminomethyl] benzamide (reference drug, MS275): To a mixture of compound 1 (1.0 mmol), 1,2-phenylenediamine $(1.2 \mathrm{mmol})$ and HBTU $(1.0 \mathrm{mmol})$ in dichloromethane $(10 \mathrm{~mL})$ was slowly added triethylamine $(4.0 \mathrm{mmol})$ cooled with ice-bath, the solution was then stirred at room temperature for $4 \mathrm{~h}$, the solid was filtered out, the residue was washed with dichloromethane twice to give the title compound. Yield: $68 \%$; mp: $157-159^{\circ} \mathrm{C}$ [14]; ${ }^{1} \mathrm{H}$ NMR (DMSO-d6): $\delta 9.62$ (s, 1H, ArCONH), 8.60 (s, 1H, N=C-H), 8.53 (d, $\mathrm{J}=4.0 \mathrm{~Hz}, 1 \mathrm{H}, \mathrm{N}=\mathrm{C}-\mathrm{H}), 7.92-7.98(\mathrm{~m}, 3 \mathrm{H}, \mathrm{Ar}-\mathrm{H}), 7.79(\mathrm{dt}, \mathrm{J}=8.0 \mathrm{~Hz}$, 1H, OCONH), 7.36-7.43 (m, 3H, Ar-H), 7.17 (d, J=12.0 Hz, 1H, Ar- 
Citation: Chen T, Jiang H, Zhou J, Li Z, Huang W, et al. (2018) Synthesis of N-Substituted Benzamide Derivatives and their Evaluation as Antitumor Agents. Med Chem (Los Angeles) 8: 273-280. doi: 10.4172/2161-0444.1000524

H), $6.97(\mathrm{t}, \mathrm{J}=16.0 \mathrm{~Hz}, 1 \mathrm{H}, \mathrm{Ar}-\mathrm{H}), 6.78(\mathrm{~d}, \mathrm{~J}=8.0 \mathrm{~Hz}, 1 \mathrm{H}, \mathrm{Ar}-\mathrm{H}), 6.60$ (t, J=12.0 Hz, 1H, Ar-H), $5.10\left(\mathrm{~s}, 2 \mathrm{H}, \mathrm{OCH}_{2}\right), 4.88\left(\mathrm{~s}, 2 \mathrm{H}, \mathrm{NH}_{2}\right), 4.28$ (d, J=8.0 Hz, 2H, $\mathrm{NCH}_{2}$ ); 13C NMR (DMSO-d6, $100 \mathrm{MHz}, \mathrm{ppm}$ ): $\delta$ $171.63,164.69,156.73,149.65,149.60,144.37,140.62,136.24,133.72$, $133.06,129.86,129.67,129.22,127.86,127.52,124.00,123.06,120.46$, $119.55,63.77,44.06$. These spectral and analytical data are as previously reported.

General procedure for the preparation of $\mathrm{N}$-substituted benzamide derivatives: To a suspension of compound $1(1.0 \mathrm{mmol})$ in anhydrous THF $(20 \mathrm{~mL})$ was added CDI $(1.2 \mathrm{mmol})$, and the mixture was stirred for $3 \mathrm{~h}$ at $60^{\circ} \mathrm{C}$. After formation of acylimidazole the clear solution was cooled to room temperature. To this solution was added different kinds of $\mathrm{NH} 2$-group contained intermediates $(1.1 \mathrm{mmol})$ and trifluoroacetic acid $(1.1 \mathrm{mmol})$, which was then stirred for $16 \mathrm{~h}$. The reaction mixture was evaporated to remove THF, and the crude product was stirred in a mixture of hexane and water $(2: 5 \mathrm{v} / \mathrm{v})$ for $1 \mathrm{~h}$, filtered and dried. The obtained solid was triturated in dichloromethane twice to afford pure title compound.

Pyridin-3-ylmethyl 4-(4-carbamoylphenylcarbamoyl) benzylcarbamate (13a): Yield: $55 \%$; $\mathrm{mp}: 213-215^{\circ} \mathrm{C}$; $\mathrm{IR}\left(\mathrm{cm}^{-1}\right): 3398$, $3313,1714,1655,1609,850,716 ;{ }^{1} \mathrm{H}$ NMR (DMSO-d $)$ ): $\delta 10.40(\mathrm{~s}, 1 \mathrm{H}$, ArCON-H), 8.61 (s, $1 \mathrm{H}, \mathrm{N}=\mathrm{C}-\mathrm{H}), 8.54(\mathrm{~d}, \mathrm{~J}=4.0 \mathrm{~Hz}, 1 \mathrm{H}, \mathrm{N}-\mathrm{CH}=\mathrm{C})$, 7.98 (t, J=8.0 Hz, 1H, OCON-H), 7.92 (d, J=8.0 Hz, 2H, Ar-H), 7.84$7.89\left(\mathrm{~m}, 4 \mathrm{H}, \mathrm{Ar}-\mathrm{H}, \mathrm{CONH}_{2}\right), 7.80(\mathrm{~d}, \mathrm{~J}=4.0 \mathrm{~Hz}, 1 \mathrm{H}, \mathrm{Ar}-\mathrm{H}), 7.57-7.64$ (t, 1H, Ar-H), 7.40-7.43 (m, 3H, Ar-H), 7.26 (s, 1H, Ar-H), $5.11(\mathrm{~s}, 2 \mathrm{H}$, $\left.\mathrm{OCH}_{2}\right), 4.29\left(\mathrm{~d}, \mathrm{~J}=4.0 \mathrm{~Hz}, 2 \mathrm{H}, \mathrm{NCH}_{2}\right) ; 13 \mathrm{C}$ NMR $(100 \mathrm{MHz}$, DMSO-d6, ppm): $\delta 167.85,166.03,156.72,149.59,144.07,142.31,136.25,133.73$, $133.15,129.56,128.68,128.31,127.34,124.01,119.76,112.90,63.73$, 44.08; ESI-HRMS: Anal. Calcd. for $\mathrm{C}_{22} \mathrm{H}_{20} \mathrm{~N}_{4} \mathrm{O}_{4}$ 13a: $\mathrm{m} / \mathrm{z}$ 404.1485; Found $\left[\mathrm{M}^{+} \mathrm{Na}\right]^{+}: \mathrm{m} / \mathrm{z} 427.1386$.

Pyridin-3-ylmethyl 4-((4-(piperidine-1-carbonyl)phenyl) carbamoyl)benzylcarbamate(13b): Yield: $50 \%$; mp: $182-185^{\circ} \mathrm{C}$; IR $\left(\mathrm{cm}^{-1}\right): 3354,3306,1724,1666,1617,764,708 ;{ }^{1} \mathrm{H}$ NMR (DMSO-d $)$ : $\delta 10.34(\mathrm{~s}, 1 \mathrm{H}, \operatorname{ArCONH}), 8.60(\mathrm{~s}, 1 \mathrm{H}, \mathrm{N}=\mathrm{C}-\mathrm{H}), 8.53(\mathrm{~d}, \mathrm{~J}=4.0 \mathrm{~Hz}$, 1H, N=C-H), 7.85-7.99 (m, 3H, Ar-H), 7.83 (d, J=4.0 Hz, 2H, Ar-H), $7.79(\mathrm{dt}, \mathrm{J}=8.0 \mathrm{~Hz}, 1 \mathrm{H}, \mathrm{OCON}-\mathrm{H}), 7.35-7.43(\mathrm{~m}, 5 \mathrm{H}, \mathrm{Ar}-\mathrm{H}), 5.10(\mathrm{~s}$, $\left.2 \mathrm{H}, \mathrm{OCH}_{2}\right), 4.28\left(\mathrm{~d}, \mathrm{~J}=8.0 \mathrm{~Hz}, 2 \mathrm{H}, \mathrm{NCH}_{2}\right), 3.43\left(\mathrm{~m}, 4 \mathrm{H}, \mathrm{CH}_{2} \mathrm{NCH}_{2}\right)$, $1.61\left(\mathrm{~m}, 2 \mathrm{H}, \mathrm{CH}_{2}\right), 1.51\left(\mathrm{~m}, 4 \mathrm{H}, \mathrm{CH}_{2}\right) ;{ }^{13} \mathrm{C}$ NMR (100 MHz, DMSO-d ppm): $\delta 169.18,165.98,156.71,149.62,149.60,144.01,140.59,136.22$, $133.80,133.13,131.79,128.27,127.96,127.34,124.00,120.16,63.73$, 44.08, 24.57; ESI-HRMS: Anal. Calcd. for $\mathrm{C}_{27} \mathrm{H}_{28} \mathrm{~N}_{4} \mathrm{O}_{4} 13 \mathrm{~b}: \mathrm{m} / \mathrm{z}$ 472.2111; Found $\left[\mathrm{M}^{+} \mathrm{Na}\right]^{+}: \mathrm{m} / \mathrm{z}$ 495.2009.

Pyridin-3-ylmethyl 4-((4-(morpholine-4-carbonyl)phenyl) carbamoyl)benzylcarbamate (13c): Yield: $52 \%$; mp: $178-180^{\circ} \mathrm{C}$; IR $\left(\mathrm{cm}^{-1}\right)^{\circ} \mathrm{C} 3361,3311,1721,1670,1612,839,760,702 ;{ }^{1} \mathrm{H}$ NMR $\left(\mathrm{DMSO}_{\mathrm{d}}\right.$ ): $\delta 10.36(\mathrm{~s}, 1 \mathrm{H}, \operatorname{ArCONH}), 8.60(\mathrm{~s}, 1 \mathrm{H}, \mathrm{N}=\mathrm{C}-\mathrm{H}), 8.53(\mathrm{~d}$, $\mathrm{J}=4.0 \mathrm{~Hz}, 1 \mathrm{H}, \mathrm{N}=\mathrm{C}-\mathrm{H}), 7.84-7.99(\mathrm{~m}, 5 \mathrm{H}, \mathrm{Ar}-\mathrm{H}), 7.79(\mathrm{dt}, \mathrm{J}=8.0 \mathrm{~Hz}$, $1 \mathrm{H}, \mathrm{OCON}-\mathrm{H}), 7.39-7.44(\mathrm{~m}, 5 \mathrm{H}, \mathrm{Ar}-\mathrm{H}), 5.10\left(\mathrm{~s}, 2 \mathrm{H}, \mathrm{OCH}_{2}\right), 4.28$ (d, J=8.0 Hz, 2H, $\left.\mathrm{NCH}_{2}\right), 3.50-3.60\left(\mathrm{~m}, 8 \mathrm{H}, \mathrm{OCH}_{2} \mathrm{CH}_{2} \mathrm{~N}\right) ;{ }^{13} \mathrm{C} \mathrm{NMR}$ (100 MHz, DMSO-d, ppm): $\delta$ 169.38, 166.02, 156.72, 149.61, 144.05, $140.94,136.22,133.76,133.13,130.74,128.44,128.29,127.34,124.00$, 120.13, 66.59, 63.73, 44.08; ESI-HRMS: Anal. Calcd. For $\mathrm{C}_{26} \mathrm{H}_{26} \mathrm{~N}_{4} \mathrm{O}_{5}$ 13c: $\mathrm{m} / \mathrm{z}$ 474.1903; Found $\left[\mathrm{M}^{+} \mathrm{Na}\right]^{+}: \mathrm{m} / \mathrm{z} 497.1800$.

Pyridin-3-ylmethyl 4-((4-(4-methylpiperazine-1-carbonyl) phenyl)carbamoyl)benzylcarbamate (13d): Yield: 48\%; $\mathrm{mp}$ : $153-$ $155^{\circ} \mathrm{C}$; IR $\left(\mathrm{cm}^{-1}\right): 3360,3303,1720,1660,845,710 ;{ }^{1} \mathrm{H}$ NMR (DMSO-d $\mathrm{d}_{6}$ : $\delta 10.38(\mathrm{~s}, 1 \mathrm{H}, \operatorname{ArCON}-\mathrm{H}), 8.61(\mathrm{~s}, 1 \mathrm{H}, \mathrm{N}=\mathrm{C}-\mathrm{H}), 8.54(\mathrm{~d}, \mathrm{~J}=4.0 \mathrm{~Hz}, 1 \mathrm{H}$, $\mathrm{N}-\mathrm{CH}=\mathrm{C}), 7.98(\mathrm{t}, \mathrm{J}=8.0 \mathrm{~Hz}, 1 \mathrm{H}, \mathrm{Ar}-\mathrm{H}), 7.91(\mathrm{~d}, \mathrm{~J}=8.0 \mathrm{~Hz}, 2 \mathrm{H}, \mathrm{Ar}-\mathrm{H})$, $7.84(\mathrm{~d}, \mathrm{~J}=12.0 \mathrm{~Hz}, 2 \mathrm{H}, \mathrm{Ar}-\mathrm{H}), 7.78$ (dt, J=8.0 Hz, 1H, OCON-H), 7.38- $7.43(\mathrm{~m}, 5 \mathrm{H}, \mathrm{Ar}-\mathrm{H}), 5.11\left(\mathrm{~s}, 2 \mathrm{H}, \mathrm{OCH}_{2}\right), 4.29$ (d, J=4.0 Hz, 2H, $\left.\mathrm{NCH}_{2}\right)$, $3.44\left(\mathrm{~m}, 4 \mathrm{H}, \mathrm{CONCH}_{2}\left(\mathrm{CH}_{2}\right)\right), 2.20\left(\mathrm{~s}, 3 \mathrm{H},-\mathrm{CH}_{3}\right), 1.23(\mathrm{t}, \mathrm{J}=8.0 \mathrm{~Hz}, 4 \mathrm{H}$, $\left.\mathrm{NCH}_{2}\left(\mathrm{CH}_{2}\right)\right) ;{ }^{13} \mathrm{C}$ NMR $\left(100 \mathrm{MHz}, \mathrm{DMSO}-\mathrm{d}_{6}, \mathrm{ppm}\right): \delta 169.25,166.00$, $156.72,149.62,149.60,144.04,140.83,136.21,133.78,133.13,131.17$, $128.29,128.26,127.34,124.00,120.15,63.73,62.99,52.47,46.09,44.08$; ESI-HRMS: Anal. Calcd. for $\mathrm{C}_{27} \mathrm{H}_{29} \mathrm{~N}_{5} \mathrm{O}_{4}$ 13d: m/z 487.2220; Found $\left[\mathrm{M}^{+} \mathrm{H}\right]^{+}: \mathrm{m} / \mathrm{z}$ 488.2295.

Pyridin-3-ylmethyl 4-((2-aminobenzoyl)carbamoyl) benzylcarbamate(13e): Yield: $46 \%$; mp: $208-210^{\circ} \mathrm{C}$; IR $\left(\mathrm{cm}^{-1}\right)$ : 3367, 3283, 1687, 1663, 764; ${ }^{1} \mathrm{H}$ NMR (DMSO-d $): \delta 12.95(\mathrm{~s}, 1 \mathrm{H}$, CONHCOAr), 8.70 (d, J=12.0 Hz, 1H, Ar-NH $), 8.61$ (s, 1H, N=C-H), $8.54(\mathrm{~d}, \mathrm{~J}=4.0 \mathrm{~Hz}, 1 \mathrm{H}, \mathrm{N}-\mathrm{CH}=\mathrm{C}), 8.43\left(\mathrm{~s}, 1 \mathrm{H}, \mathrm{Ar}-\mathrm{NH}_{2}\right), 7.98$ (t, J=8.0 $\mathrm{Hz}, 1 \mathrm{H}, \mathrm{OCON}-\mathrm{H}$ ), 7.89-7.93 (m, 3H, Ar-H), 7.85 (s, 1H, Ar-H), 7.81 $(\mathrm{d}, \mathrm{J}=8.0 \mathrm{~Hz}, 1 \mathrm{H}, \mathrm{Ar}-\mathrm{H}), 7.58(\mathrm{t}, \mathrm{J}=8.0 \mathrm{~Hz}, 1 \mathrm{H}, \mathrm{Ar}-\mathrm{H}), 7.41-7.46(\mathrm{~m}$, $3 \mathrm{H}, \mathrm{Ar}-\mathrm{H}), 7.18(\mathrm{t}, \mathrm{J}=8.0 \mathrm{~Hz}, 1 \mathrm{H}, \mathrm{Ar}-\mathrm{H}), 5.12\left(\mathrm{~s}, 2 \mathrm{H}, \mathrm{OCH}_{2}\right), 4.30(\mathrm{~d}$, $\left.\mathrm{J}=4.0 \mathrm{~Hz}, 2 \mathrm{H}, \mathrm{NCH}_{2}\right) ;{ }^{13} \mathrm{C}$ NMR $\left(100 \mathrm{MHz}, \mathrm{DMSO}-\mathrm{d}_{6}, \mathrm{ppm}\right): \delta 171.64$, $164.69,156.74,149.65,149.60,144.37,140.62,136.24,133.73,133.12$, $133.05,129.22,127.86,127.52,124.00,123.06,120.48,119.56,63.78$, 44.07; ESI-HRMS: Anal. Calcd. for $\mathrm{C}_{22} \mathrm{H}_{20} \mathrm{~N}_{4} \mathrm{O}_{4}$ 13e: $\mathrm{m} / \mathrm{z} 404.1485$. Found $\left[\mathrm{M}^{+} \mathrm{Na}\right]^{+}: \mathrm{m} / \mathrm{z} 427.1383$.

Pyridin-3-ylmethyl 4-(2-(2-aminobenzoyl) hydrazinecarbonyl) benzylcarbamate (13f): Yield: $43 \%$; $\mathrm{mp}: 156-158^{\circ} \mathrm{C}$; IR $\left(\mathrm{cm}^{-1}\right): 3489$, $3361,3294,3244,1726,1677,1615,823,753 ;{ }^{1} \mathrm{H}$ NMR (DMSO-d $)$ ): $\delta$ 10.34 (s, 1H, ArCONHNHCO), 10.15 (s, 1H, ArCONHNHCO), 8.61 $(\mathrm{s}, 1 \mathrm{H}, \mathrm{N}=\mathrm{C}-\mathrm{H}), 8.54(\mathrm{~d}, \mathrm{~J}=4.0 \mathrm{~Hz}, 1 \mathrm{H}, \mathrm{N}-\mathrm{CH}=\mathrm{C}), 7.96(\mathrm{t}, \mathrm{J}=4.0,8.0$ $\mathrm{Hz}, 1 \mathrm{H}, \mathrm{Ar}-\mathrm{H}), 7.87$ (d, J=8.0 Hz, 2H, Ar-H), 7.78 (d, J=8.0 Hz, 1H, OCONH), $7.61(\mathrm{~d}, \mathrm{~J}=8.0 \mathrm{~Hz}, 1 \mathrm{H}, \mathrm{Ar}-\mathrm{H}), 7.37-7.43(\mathrm{~m}, 3 \mathrm{H}, \mathrm{Ar}-\mathrm{H})$, $7.20(\mathrm{~m}, 1 \mathrm{H}, \mathrm{Ar}-\mathrm{H}), 6.76(\mathrm{~d}, \mathrm{~J}=8.0 \mathrm{~Hz}, 1 \mathrm{H}, \mathrm{Ar}-\mathrm{H}), 6.53(\mathrm{~m}, 1 \mathrm{H}, \mathrm{Ar}-$ $\mathrm{H}), 6.43\left(\mathrm{~s}, 2 \mathrm{H}, \mathrm{Ar}-\mathrm{NH}_{2}\right), 5.11\left(\mathrm{~s}, 2 \mathrm{H}, \mathrm{OCH}_{2}\right), 4.28(\mathrm{~d}, \mathrm{~J}=4.0 \mathrm{~Hz}, 2 \mathrm{H}$, $\left.\mathrm{NCH}_{2}\right) ;{ }^{13} \mathrm{C}$ NMR $(100 \mathrm{MHz}$, DMSO-d, $\mathrm{ppm}): \delta 168.78,166.23,156.74$, $150.38,149.63,149.59,144.04,136.22,133.13,132.76,131.73,128.64$, 128.00, 127.39, 124.00, 116.89, 115.09, 113.04, 63.76, 44.09. ESI-HRMS: Anal. Calcd. for $\mathrm{C}_{22} \mathrm{H}_{21} \mathrm{~N}_{5} \mathrm{O}_{4}$ 13f: $\mathrm{m} / \mathrm{z}$ 419.1594. Found $\left[\mathrm{M}^{+} \mathrm{Na}\right]^{+}: \mathrm{m} / \mathrm{z}$ 442.1493 .

Pyridin-3-ylmethyl

4-(2-(2-amino-4-chlorobenzoyl) hydrazinecarbonyl)benzylcarbamate (13g): Yield: 49\%; $\mathrm{mp}$ : 204-206 ${ }^{\circ} \mathrm{C}$; IR $\left(\mathrm{cm}^{-1}\right): 3404,3340,3292,1708,1682,1642,718 ;{ }^{1} \mathrm{H}$ NMR (DMSO-d $): \delta 10.37$ (s, 1H, ArCONHNHCO), 10.24 (s, 1H, ArCONHNHCO), $8.60(\mathrm{~s}, 1 \mathrm{H}, \mathrm{N}=\mathrm{C}-\mathrm{H}), 8.54(\mathrm{~d}, \mathrm{~J}=4.0 \mathrm{~Hz}, 1 \mathrm{H}$, $\mathrm{N}-\mathrm{CH}=\mathrm{C}), 7.96(\mathrm{t}, \mathrm{J}=4.0,8.0 \mathrm{~Hz}, 1 \mathrm{H}, \mathrm{Ar}-\mathrm{H}), 7.88(\mathrm{~d}, \mathrm{~J}=8.0 \mathrm{~Hz}, 2 \mathrm{H}, \mathrm{Ar}-$ $\mathrm{H}), 7.78(\mathrm{~d}, \mathrm{~J}=8.0 \mathrm{~Hz}, 1 \mathrm{H}, \mathrm{OCONH}), 7.61(\mathrm{~d}, \mathrm{~J}=8.0 \mathrm{~Hz}, 1 \mathrm{H}, \mathrm{Ar}-\mathrm{H})$, 7.37-7.43 (m, 3H, Ar-H), $6.82(\mathrm{~s}, 1 \mathrm{H}, \mathrm{Ar}-\mathrm{H}), 6.69$ (s, 2H, Ar- $\left.\mathrm{NH}_{2}\right), 6.57$ $(\mathrm{d}, \mathrm{J}=8.0 \mathrm{~Hz}, 1 \mathrm{H}, \mathrm{Ar}-\mathrm{H}), 5.11\left(\mathrm{~s}, 2 \mathrm{H}, \mathrm{OCH}_{2}\right), 4.28(\mathrm{~d}, \mathrm{~J}=4.0 \mathrm{~Hz}, 2 \mathrm{H}$, $\left.\mathrm{NCH}_{2}\right) ;{ }^{13} \mathrm{C}$ NMR $\left(100 \mathrm{MHz}\right.$, DMSO-d $\left.\mathrm{d}_{6} \mathrm{ppm}\right): \delta 168.01,166.23,156.73$, $151.67,149.62,149.59,144.10,137.29,136.21,133.12,131.62,130.46$, 128.00, 127.40, 123.99, 115.65, 114.82, 111.77, 63.76, 44.08. ESI-HRMS: Anal. Calcd. for $\mathrm{C}_{22} \mathrm{H}_{20} \mathrm{ClN}_{5} \mathrm{O}_{4} 13 \mathrm{~g}: \mathrm{m} / \mathrm{z} 453.1204$. Found $\left[\mathrm{M}^{+} \mathrm{Na}\right]^{+}: \mathrm{m} / \mathrm{z}$ 476.1108 .

Pyridin-3-ylmethyl 4-(2-(2-hydroxybenzoyl)hydrazinecarbonyl) benzylcarbamate (13h): Yield: $43 \%$; mp: $172-174^{\circ} \mathrm{C}$; IR $\left(\mathrm{cm}^{-1}\right): 3301$, 1720, 1607, 802, 759; ${ }^{1} \mathrm{H}$ NMR (DMSO-d $)$ ): $\delta 11.95$ (s, $1 \mathrm{H}, \mathrm{Ar}-\mathrm{OH}$ ), $10.68(\mathrm{~s}, 1 \mathrm{H}, \mathrm{ArCONHNHCO}), 10.64(\mathrm{~s}, 1 \mathrm{H}, \mathrm{ArCONHNHCO}), 8.60$ $(\mathrm{s}, 1 \mathrm{H}, \mathrm{N}=\mathrm{C}-\mathrm{H}), 8.54(\mathrm{~d}, \mathrm{~J}=4.0 \mathrm{~Hz}, 1 \mathrm{H}, \mathrm{N}-\mathrm{CH}=\mathrm{C}), 7.93-7.98(\mathrm{~m}, 2 \mathrm{H}$, Ar-H), 7.88 (d, J=8.0 Hz, 2H, Ar-H), 7.78-7.81 (m,1H, Ar-H), 7.38-7.49 (m, 4H, Ar-H), 6.94-7.00 (m, 2H, Ar-H), $5.11\left(\mathrm{~s}, 2 \mathrm{H}, \mathrm{OCH}_{2}\right), 4.28(\mathrm{~d}$ $\left.\mathrm{J}=4.0 \mathrm{~Hz}, 2 \mathrm{H}, \mathrm{NCH}_{2}\right) ;{ }^{13} \mathrm{C}$ NMR $\left(100 \mathrm{MHz}, \mathrm{DMSO}-\mathrm{d}_{6}, \mathrm{ppm}\right): \delta 168.21$, $165.82,159.77,156.73,149.62,149.59,144.25,136.22,134.64,133.12$, $131.35,128.74,128.06,127.44,124.00,119.53,117.87,115.01,63.76$, 44.09. ESI-HRMS: Anal. Calcd. for $\mathrm{C}_{22} \mathrm{H}_{20} \mathrm{~N}_{4} \mathrm{O}_{5}$ 13h: m/z 420.1434 
Citation: Chen T, Jiang H, Zhou J, Li Z, Huang W, et al. (2018) Synthesis of N-Substituted Benzamide Derivatives and their Evaluation as Antitumor Agents. Med Chem (Los Angeles) 8: 273-280. doi: 10.4172/2161-0444.1000524

Found $\left[\mathrm{M}^{+} \mathrm{Na}\right]^{+}: \mathrm{m} / \mathrm{z} 443.1328$.

Pyridin-3-ylmethyl

4-(2-(5-chloro-2-hydroxybenzoyl) hydrazinecarbonyl)benzylcarbamate (13i): Light yellow solid; Yield: 47\%; mp: 212-214 ${ }^{\circ} \mathrm{C}$; IR $\left(\mathrm{cm}^{-1}\right)$ : 3303, 1719, 1601, 1560, 706; ${ }^{1} \mathrm{H}$ NMR (DMSO-d $\mathrm{d}_{6}: \delta 11.93(\mathrm{~s}, 1 \mathrm{H}, \mathrm{Ar}-\mathrm{OH}), 10.72(\mathrm{~s}, 2 \mathrm{H}, \mathrm{ArCONHNHCO})$, $8.61(\mathrm{~s}, 1 \mathrm{H}, \mathrm{N}=\mathrm{C}-\mathrm{H}), 8.54(\mathrm{~d}, \mathrm{~J}=4.0 \mathrm{~Hz}, 1 \mathrm{H}, \mathrm{N}-\mathrm{CH}=\mathrm{C}), 7.95-7.98(\mathrm{~m}$, 2H, Ar-H), 7.88 (d, J=8.0 Hz, 2H, Ar-H), 7.78-7.81 (m, 2H, Ar-H), 7.50 (dd, J=4.0, $8.0 \mathrm{~Hz}, 1 \mathrm{H}, \mathrm{Ar}-\mathrm{H}), 7.39-7.43$ (m, 3H, Ar-H), 7.03 (d, J=8.0 $\mathrm{Hz}, 1 \mathrm{H}, \mathrm{Ar}-\mathrm{H}), 5.11\left(\mathrm{~s}, 2 \mathrm{H}, \mathrm{OCH}_{2}\right), 4.29\left(\mathrm{~d}, \mathrm{~J}=4.0 \mathrm{~Hz}, 2 \mathrm{H}, \mathrm{NCH}_{2}\right) ;{ }^{13} \mathrm{C}$ NMR (100 MHz, DMSO-d 6 , ppm): $\delta 166.38,165.68,157.97,156.73$, $149.62,149.59,144.29,136.22,134.07,133.12,131.25,128.43,128.09$, 127.44, 123.99, 123.22, 119.78, 117.13, 63.76, 44.09. ESI-HRMS: Anal. Calcd. for $\mathrm{C}_{22} \mathrm{H}_{19} \mathrm{ClN}_{4} \mathrm{O}_{5}$ 13i: $\mathrm{m} / \mathrm{z}$ 454.1044. Found $\left[\mathrm{M}^{+} \mathrm{Na}\right]^{+}: \mathrm{m} / \mathrm{z}$ 477.0942 .

\section{Pyridin-3-ylmethyl}

4-(2-nicotinoylhydrazinecarbonyl) benzylcarbamate(13j): Yield: $45 \%$; $\mathrm{mp}: 116-118^{\circ} \mathrm{C}$; IR $\left(\mathrm{cm}^{-1}\right): 3426$, $3322,3287,1699,1650,709 ;{ }^{1} \mathrm{H}$ NMR $\left(\right.$ DMSO-d $\left._{6}\right): \delta 10.71(\mathrm{~s}, 1 \mathrm{H}$, ArCONHNHCO), 10.58 (s, 1H, ArCONHNHCO), 9.08 (s, J=4.0 Hz, $1 \mathrm{H}, \mathrm{Ar}-\mathrm{H}), 8.78$ (dd, J=4.0, $8.0 \mathrm{~Hz}, 1 \mathrm{H}, \mathrm{Ar}-\mathrm{H}), 8.60$ (s, 1H, N=C-H), $8.54(\mathrm{~d}, \mathrm{~J}=4.0 \mathrm{~Hz}, 1 \mathrm{H}, \mathrm{N}-\mathrm{CH}=\mathrm{C}), 8.25-8.28(\mathrm{~m}, 1 \mathrm{H}, \mathrm{Ar}-\mathrm{H}), 7.96(\mathrm{t}, \mathrm{J}=4.0$, $8.0 \mathrm{~Hz}, 1 \mathrm{H}, \mathrm{Ar}-\mathrm{H}), 7.88$ (d, J=8.0 Hz, 2H, Ar-H), 7.78 (m, 1H, Ar-H), 7.56-7.60 (m, 1H, Ar-H), 7.39-7.43 (m, 3H, Ar-H), $5.11\left(\mathrm{~s}, 2 \mathrm{H}, \mathrm{OCH}_{2}\right)$, $4.29\left(\mathrm{~d}, \mathrm{~J}=4.0 \mathrm{~Hz}, 2 \mathrm{H}, \mathrm{NCH}_{2}\right) ;{ }^{13} \mathrm{C}$ NMR $\left(100 \mathrm{MHz}, \mathrm{DMSO}-\mathrm{d}_{6}, \mathrm{ppm}\right)$ : $\delta 166.10,164.95,156.74,152.99,149.62,149.59,148.89,144.23,136.22$, $135.68,133.12,131.47,128.71,128.03,127.46,124.19,124.00,63.76$, 44.08. ESI-HRMS: Anal. Calcd. for $\mathrm{C}_{21} \mathrm{H}_{19} \mathrm{~N}_{5} \mathrm{O}_{4}$ 13j: $\mathrm{m} / \mathrm{z} 4005.1437$. Found $\left[\mathrm{M}^{+} \mathrm{H}\right]^{+}: \mathrm{m} / \mathrm{z} 406.1511$.

Pyridin-3-ylmethyl

4-(2-picolinoylhydrazinecarbonyl) benzylcarbamate (13k): Yield: 41\%; mp: $178-180^{\circ} \mathrm{C}$; IR $\left(\mathrm{cm}^{-1}\right)$ : $3312,1702,1657,1531,712 ;{ }^{1} \mathrm{H}$ NMR (DMSO-d $): \delta 10.62(\mathrm{~s}, 1 \mathrm{H}$, CONHNHCO), $10.53(\mathrm{~s}, 1 \mathrm{H}, \mathrm{CONHNHCO}), 8.71(\mathrm{dt}, \mathrm{J}=4.0 \mathrm{~Hz}, 1 \mathrm{H}$, Ar-H), 8.61 (s, 1H, N=C-H), 8.54 (d, J=4.0 Hz, 1H, N-CH=C), 8.02$8.08(\mathrm{~m}, 2 \mathrm{H}, \mathrm{Ar}-\mathrm{H}), 7.97(\mathrm{t}, \mathrm{J}=4.0,8.0 \mathrm{~Hz}, 1 \mathrm{H}, \mathrm{Ar}-\mathrm{H}), 7.88(\mathrm{~d}, \mathrm{~J}=8.0$ $\mathrm{Hz}, 2 \mathrm{H}, \mathrm{Ar}-\mathrm{H}), 7.79$ (m, 1H, Ar-H), 7.65-7.68 (m, 1H, Ar-H), 7.38-7.43 $(\mathrm{m}, 3 \mathrm{H}, \mathrm{Ar}-\mathrm{H}), 5.12\left(\mathrm{~s}, 2 \mathrm{H}, \mathrm{OCH}_{2}\right), 4.29\left(\mathrm{~d}, \mathrm{~J}=4.0 \mathrm{~Hz}, 2 \mathrm{H}, \mathrm{NCH}_{2}\right) ;{ }^{13} \mathrm{C}$ NMR (100 MHz, DMSO-d, ppm): $\delta 165.73,163.71,156.75,149.75$, $149.62,149.59,149.15,144.07,138.32,136.22,133.13,131.62,128.06$, 127.46, 127.37, 124.00, 122.84, 63.76, 44.09. ESI-HRMS: Anal. Calcd. for $\mathrm{C}_{21} \mathrm{H}_{19} \mathrm{~N}_{5} \mathrm{O}_{4} 13 \mathrm{k}: \mathrm{m} / \mathrm{z}$ 4005.1437. Found $\left[\mathrm{M}^{+} \mathrm{H}\right]^{+}: \mathrm{m} / \mathrm{z} 406.1508$.

Pyridin-3-ylmethyl 4-((2-fluoro-4-(piperidin-1-yl)phenyl) carbamoyl)benzylcarbamate(13l): Gray solid; Yield: 45\%; mp: 176 $178^{\circ} \mathrm{C}$; IR $\left(\mathrm{cm}^{-1}\right): 3362,864,712 ;{ }_{1} \mathrm{H}$ NMR (DMSO-d $\left.\mathrm{d}_{6}\right): \delta 10.21(\mathrm{~s}, 1 \mathrm{H}$, $\operatorname{ArCONH}), 8.60$ (s, $1 \mathrm{H}, \mathrm{N}=\mathrm{C}-\mathrm{H}), 8.53(\mathrm{~d}, \mathrm{~J}=4.0 \mathrm{~Hz}, 1 \mathrm{H}, \mathrm{N}-\mathrm{CH}=\mathrm{C})$, 7.89-7.97 (m, 3H, Ar-H), $7.80(\mathrm{~d}, \mathrm{~J}=8.0 \mathrm{~Hz}, 1 \mathrm{H}, \mathrm{OCONH}), 7.69$ (dd, $\mathrm{J}=4.0,16.0 \mathrm{~Hz}, 1 \mathrm{H}, \mathrm{Ar}-\mathrm{H}), 7.47$ (dd, J=4.0, $8.0 \mathrm{~Hz}, 1 \mathrm{H}$, Ar-H), 7.38$7.43(\mathrm{~m}, 3 \mathrm{H}, \mathrm{Ar}-\mathrm{H}), 7.01(\mathrm{t}, \mathrm{J}=8.0,12.0 \mathrm{~Hz}, 1 \mathrm{H}, \mathrm{Ar}-\mathrm{H}), 5.10(\mathrm{~s}, 2 \mathrm{H}$, $\left.\mathrm{OCH}_{2}\right), 4.28\left(\mathrm{~d}, \mathrm{~J}=4.0 \mathrm{~Hz}, 2 \mathrm{H}, \mathrm{NCH}_{2}\right), 3.74\left(\mathrm{~m}, 4 \mathrm{H}, \mathrm{CH}_{2} \mathrm{OCH}_{2}\right), 2.97$ (m, $\left.4 \mathrm{H}, \mathrm{CH}_{2} \mathrm{NCH}_{2}\right) ;{ }^{13} \mathrm{C}$ NMR (100 MHz, DMSO-d, $\left.\mathrm{ppm}\right): \delta 165.50$, $156.72,149.61,143.84,136.20,134.41,134.30,133.82,133.14,128.13$, $127.32,123.98,119.74,119.70,116.69,108.98,108.73,63.74,52.22$, 44.09, 26.21, 24.21. ESI-HRMS: Anal. Calcd. for $\mathrm{C}_{26} \mathrm{H}_{27} \mathrm{FN}_{4} \mathrm{O}_{3} 13 \mathrm{l}: \mathrm{m} / \mathrm{z}$ 462.2067. Found $\left[\mathrm{M}^{+} \mathrm{H}\right]^{+}: \mathrm{m} / \mathrm{z} 463.2140$.

Pyridin-3-ylmethyl 4-((2-fluoro-4-morpholinophenyl) carbamoyl) benzylcarbamate $(13 \mathrm{~m})$ : Yield: $46 \%$; mp: $180-182^{\circ} \mathrm{C}$; IR $\left(\mathrm{cm}^{-1}\right): 3337,1695,1634,754,719 ;{ }^{1} \mathrm{H}$ NMR (DMSO-d $): \delta 10.18(\mathrm{~s}$, $1 \mathrm{H}, \operatorname{ArCONH}), 8.60(\mathrm{~s}, 1 \mathrm{H}, \mathrm{N}=\mathrm{C}-\mathrm{H}), 8.54(\mathrm{~d}, \mathrm{~J}=4.0 \mathrm{~Hz}, 1 \mathrm{H}, \mathrm{N}-\mathrm{CH}=\mathrm{C})$, 7.88-7.98 (m, 3H, Ar-H), $7.80(\mathrm{~d}, \mathrm{~J}=8.0 \mathrm{~Hz}, 1 \mathrm{H}, \mathrm{OCONH}), 7.69(\mathrm{dd}$, $\mathrm{J}=4.0,16.0 \mathrm{~Hz}, 1 \mathrm{H}, \mathrm{Ar}-\mathrm{H}), 7.38-7.46(\mathrm{~m}, 4 \mathrm{H}, \mathrm{Ar}-\mathrm{H}), 7.01$ (t, J=8.0, 12.0 $\mathrm{Hz}, 1 \mathrm{H}, \mathrm{Ar}-\mathrm{H}), 5.10\left(\mathrm{~s}, 2 \mathrm{H}, \mathrm{OCH}_{2}\right), 4.28$ (d, J=4.0 Hz, 2H, $\left.\mathrm{NCH}_{2}\right), 2.92$ (m, 4H, $\left.\mathrm{CH}_{2} \mathrm{NCH}_{2}\right), 1.63-1.66\left(\mathrm{~m}, 4 \mathrm{H}, \mathrm{NCH}_{2} \mathrm{CH}_{2}\right) ; 1.50\left(\mathrm{~m}, 2 \mathrm{H}, \mathrm{CH}_{2}\right)$; ${ }^{13} \mathrm{C}$ NMR (100 MHz, DMSO-d 6 , ppm): $\delta 165.55,156.72,149.59,143.89$, $136.20,134.83,134.72,133.76,133.13,128.15,127.33,123.98,119.42$ 119.37, 116.73, 109.06, 108.81, 66.67, 63.74, 51.21, 44.09. ESI-HRMS: Anal. Calcd. for $\mathrm{C}_{25} \mathrm{H}_{25} \mathrm{FN}_{4} \mathrm{O}_{4} 13 \mathrm{~m}: \mathrm{m} / \mathrm{z}$ 464.1860. Found $\left[\mathrm{M}^{+} \mathrm{H}\right]^{+}: \mathrm{m} / \mathrm{z}$ 465.1934.

Pyridin-3-ylmethyl 4-((2-hydroxy-5-nitrophenyl) carbamoyl) benzylcarbamate (13n): Yellow solid; Yield: $43 \%$; mp: $178-180^{\circ} \mathrm{C}$ IR $\left(\mathrm{cm}^{-1}\right): 3400,3332,1705,1656,1595,749 ;{ }^{1} \mathrm{H}$ NMR (DMSO-d $)$ : $\delta$ 11.65 (s, 1H, Ar-OH), 9.58 (s, 1H, ArCONH), 8.80 (d, J=4.0 Hz, $1 \mathrm{H}$, Ar-H), 8.61 (s, 1H, N=C-H), 8.54 (d, J=4.0 Hz, 1H, N-CH=C), 7.948.02 (m, 4H, Ar-H), 7.80 (m, 1H, Ar-H), 7.40-7.43 (m, 3H, Ar-H), 7.08 (d, J=12.0 Hz, 1H, Ar-H), $5.12\left(\mathrm{~s}, 2 \mathrm{H}, \mathrm{OCH}_{2}\right), 4.30(\mathrm{~d}, \mathrm{~J}=4.0 \mathrm{~Hz}, 2 \mathrm{H}$, $\left.\mathrm{NCH}_{2}\right) ;{ }^{13} \mathrm{C} \mathrm{NMR}(100 \mathrm{MHz}$, DMSO-d, $\mathrm{ppm}): \delta 165.69,156.73,156.13$, $149.62,149.58,144.34,139.54,136.23,133.13,132.98,128.18,127.51$, $126.74,124.00,122.17,119.23,115.69,63.76,56.51,44.09,19.02$. ESIHRMS: Anal. Calcd. for $\mathrm{C}_{21} \mathrm{H}_{18} \mathrm{~N}_{4} \mathrm{O}_{6} 13 \mathrm{n}: \mathrm{m} / \mathrm{z} 422.1226$. Found $\left[\mathrm{M}^{+} \mathrm{H}\right]^{+}$: $\mathrm{m} / \mathrm{z} 423.1300$.

\section{Biology}

\section{Antitumor activity test}

The antitumor activity of all the synthesized compounds (13a-n) against cancer cell lines of MCF-7, A549, K562 and MDA-MB-231 by the MTT assay was carried out in the State Key Laboratory of Biotherapy, Sichuan University. All the data were obtained by the mean values of three repeated experiments. Briefly, Cells $\left(3 \times 10^{4} / \mathrm{ml}\right)$ were seeded in 96-well plates and cultured for 24 hours, followed by various concentration of compounds from $50 \mu \mathrm{M}$ to $1.56 \mu \mathrm{M}$ treatment for 48 hours at $37^{\circ} \mathrm{C}, 5 \% \mathrm{CO}_{2}$. Then, $20 \mu \mathrm{l}$ of $5 \mathrm{mg} / \mathrm{ml}$ MTT was added per well and incubated for another 4 hours at $37^{\circ} \mathrm{C}$, the supernatant fluid was removed, and DMSO was added $150 \mu \mathrm{l} /$ well for 15-20 minutes. The light absorptions (OD) were measured at $570 \mathrm{~nm}$ with SpectraMAX M5 microplate spectrophotometer (Molecular Devices). Entinostat was used as referential drug for anti-proliferative evaluation, respectively, which was also assessed under similar conditions for comparison with the tested compounds. The response parameter calculated was the $\mathrm{IC}_{50}$ value, which corresponds to the concentration required for $50 \%$ inhibition of cell viability. The data for the anti-proliferative activity are presented in Table 1.

\section{Results and Discussion}

\section{Chemistry}

The synthetic strategy of $\mathrm{N}$-substituted benzamide derivatives was depicted in Schemes 1-5. Compound 1 was obtained by condensation of pyridin-3-ylmethanol and 4-(aminomethyl) benzoic acid in the presence of CDI in dry THF with $83 \%$ yield (Scheme 1), which was used as the starting material to react with amino-containing intermediates. Three kinds of amino-containing intermediates including substituted anilines, substituted aromatic amides and substituted aromatic hydrazides were synthesized. 4-Nitrobenzoic acid was transferred into acyl chloride in $\mathrm{SOCl}_{2}$ and then reacted with different kinds of amines in the presence of triethylamine to abtain $2 \mathrm{a}-\mathrm{d}$, which was then reduced under the condition of $\mathrm{H}_{2} / \mathrm{Pd}-\mathrm{C}$ to give $3 \mathrm{a}-\mathrm{d}$ (Scheme 2). Substituted aromatic amides $5 \mathrm{a}-\mathrm{b}, 8 \mathrm{a}-\mathrm{b}$ and substituted aromatic hydrazides 7a-b, 9a-b and 10a-b can be formed from methyl benzoate reacted with ammonia and hydrazine hydrate, respectively. Nitro group compounds were then reduced to amino group in the presence of $\mathrm{Fe} /$ $\mathrm{NH} \mathrm{Cl}$ (Scheme 3). 2,4-Difluoronitrobenzene was firstly reacted with cyclic amines to afford 11a-b, which were then similarly reduced to give 
12a-b (Scheme 4). Compound 1 was condensed with $\mathrm{NH}_{2}$-contained intermediates in the presence of CDI in dry THF to form the target compounds (Scheme 5) with a moderate yield.

\section{Biology}

The anti-proliferative activities in vitro of compounds 13a-n were evaluated by MTT assay and Entinostat (MS-275) as the referential compound. Four different cancer cell lines which are human breast adenocarcinoma cell (MCF-7), human lung cell (A549), Human chronic myeloid leukemia cell (K562) and human breast cancer cell (MAD-MB-231) were chosen to be tested. The results are shown in Table 1.

As can be seen, $13 \mathrm{~h}$ displayed good inhibitory activity against all four cell lines, even better activity than MS-275 towards A549; 13d, 13e, 13f, 13k also showed good inhibitory activity against A549 compared with MS-275; 13e, 13l, 13m displayed the similar activity against K562 compared with MS-275, 13f, 13k showed good activity against K562; $13 \mathrm{i}$ and $13 \mathrm{k}$ exhibited moderate activity against MCF-7; 13a, 13h and $13 \mathrm{i}$ also displayed moderate activity against MDA-MB-231. Other compounds showed low or no inhibitory activity towards the cancer cell lines.

Among the data reveal that, as for the zinc binding group, 2- $\mathrm{NH}_{2}$ or $2-\mathrm{OH}$ on phenyl group of $\mathrm{R}$ part is necessary for the inhibitory activity, i.e., generally $13 \mathrm{a}-\mathrm{d}$ vs $13 \mathrm{e}-\mathrm{i}$, while chlorine atom existed in the same benzene ring will largely decrease their activity, i.e., $13 \mathrm{f}$ vs $13 \mathrm{~g}$ and $13 \mathrm{~h}$ vs $13 \mathrm{i}$, the same function seen in nitro-group (13n vs MS275). Compounds containing $2-\mathrm{F}$ and 4 -cyclic amine on phenyl group of $\mathrm{R}$ part (131 and $13 \mathrm{~m}$ ) displayed good inhibitory activity towards K562. Hydrazide or bisamide fragments rarely influence the results, i.e., $13 \mathrm{e}$ vs $13 \mathrm{f}$. What's more, it seems that picolinic structure could also bind with zinc ion to exert their biological activity, like 13k displayed; however, 13j lacked activity.

\section{Molecular docking study}

In order to achieve the binding modes of the synthesized compounds and provide more insights into the interactions, a molecular docking simulation of the compounds $13 \mathrm{e}, 13 \mathrm{f}, 13 \mathrm{~h}, 13 \mathrm{k}$ and MS-275 which displayed low IC50 values was performed using Auto dock Vina (ver.1.1.2). HDAC2 and HDAC8 were chosen as the targets, their structures were prepared for docking as described previously $[12,13]$. Polar hydrogen's were added, ensuring that the protonation states were correct, and AMBER charges were assigned to receptor atoms. The ligands designed as reference ligands henceforth were extracted separately from the proteins. All water molecules were removed from the structures. The binding site was defined using the reference ligands, and all protein atoms within the geometrical constrain of $8 \AA$ of a non-hydrogen atom in any of the reference ligands were included. The structures of HDAC proteins outside of active sites remained unchanged, while active sites were kept partially flexible upon docking. Binding affinity was computed from contributions of internal and external van der Waals (vdW), hydrophobic properties (HP) and hydrogen bond (HB) using Gold score (empirical scoring) function. Finally, binding affinity and bioactivity of ligands were evaluated by calculated fitness. The results are shown in Table 2.

As can be seen, fitness value (the higher the value is, the stronger the interaction between a ligand and a protein is) is quite close for above four compounds (also close to the value of MS-275), i.e., the interaction (binding affinity) between the ligand and the protein is similar. Below is the docking modes of $13 \mathrm{~h}$ and $13 \mathrm{k}$ to HDAC2 respectively (Figure 2). The hydrogen bonds (HB) are shown in dotted yellow lines with $\mathrm{HB}$ distance of 2.45 2.97 $\AA$ and important residues are shown and labeled. Besides hydrogen bonds shown above, short contacts such as Van der Waals bond and hydrophobic interactions are also found between $13 \mathrm{~h}$ and HDAC2 (residues HIS180, GLY306 and PHE 210), the same as $13 \mathrm{k}$. These docking results are in agreement with those determined data.

\section{Acknowledgments}

We thank State Key Laboratory of Biotherapy, West China Hospital, Sichuan University for ${ }^{1} \mathrm{H}$ NMR, ${ }^{13} \mathrm{C}$ NMR and HRMS determination. We thank Engineering Teaching Experiment Center, School of Chemical Engineering for IR determination. We especially thank Assoc. Prof. Xiang Gao for finishing the molecular docking study.

\section{References}

1. Venugopal B, Evans TRJ (2011) Developing histone deacetylase inhibitors as anti-cancer therapeutics. Cur Med Chem 18: 1658-1671.

2. Kim HJ, Bae SC (2011) Histone deacetylase inhibitors: molecular mechanisms of action and clinical trials as anti-cancer drugs. Am J Transl Res 3: 166-179.

3. Gao S, Zang J, Gao QW, Liang XW, Ding QG, et al. (2017) Design, synthesis and anti-tumor activity study of novel histone deacetylase inhibitors containing isatin-based caps and O-phenylenediamine-based zinc binding groups. Bioorg Med Chem 25: 2981-2994.

4. Ellis L, Pili R (2010) Histone deacetylase inhibitors: advancing therapeutic strategies in hematological and solid malignancies. Pharma 3: 2441-2469.

5. Petrella A, Fontanella B, Carratu A, Bizzarro V, Rodriquez M, et al. (2011) Histone deacetylase inhibitors in the treatment of hematological malignancies. Mini-Revs Med Chem 11: 519- 527.

6. Lu H, Chen YD, Yang B, You QD (2011) Design, synthesis and biologica evaluation of novel histone deacetylase inhibitors based on virtual screening Acta Pharmacol Sin B 1: 240-247.

7. Wang HS, Dymock BW (2009) New patented histone deacetylase inhibitors Expert Opin Ther Patents 19: 1727-1757.

8. Sonnemann J, Marx C, Becker S, Wittig S, Palani CD, et al. (2014) P53dependent and p53-independent anticancer effects of different histone deacetylase inhibitors. Brit J Cancer 110: 656-667.

9. Mai A, Massa S, Rotili D (2006) Synthesis and biological properties of novel, uracil-containinghistone deacetylase inhibitors [J]. J Med Chem 49: 60466056.

10. Zhang X, Lv PC, Li DD, Zhang WM, Zhu HL (2015) Navigating into the chemical space between MGCD0103 and SAHA: novel histone ceacetylase inhibitors as a promising lead. Med Chem Comm 6: 1816-1825.

11. Cai JZ, Zhang QW, Lin KZ, Hu LF, Zheng YC (2015) The effect of MGCD0103 on CYP450 isoforms activity of rats by cocktail method. Bio Med Res Inter 51: 295.

12. Babaoglu K, Simeonov A, Irwin JJ, Nelson ME, Feng B, et al. (2008) Comprehensive mechanistic analysis of hits from high-throughput and docking screens against b-lactamase. J Med Chem 51: 2502-2511.

13. Irwin JJ, Raushel FM, Shoichet B (2005) Virtual screening against metalloenzymes for inhibitors and substrates. Biochemistry 44: 12316-12328.

14. Saito A, Mariko Y, Yamashita T, Nakanishi O (1999) Synthesis and Histone Deacetylase Inhibitory Activity of New Benzamide Derivatives. J Med Chem 42: 3001-3003

15. Dighe SU, Batra S (2016) Visible light-induced iodine-catalyzed transformation of terminal alkynes to primary amides via $\mathrm{C} \equiv \mathrm{C}$ bond cleavage under aqueous conditions. Adv Synth Cat 358: 500-505.

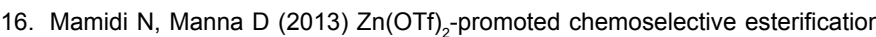
of hydroxyl group bearing carboxylic acids. J Org Chem 78: 2386-2396.

17. Gu JJ, Fang Z, Liu CK, Li X, Wei P, et al. (2016) Direct oxidative amination of aromatic aldehydes with amines in a continuous flow system using a metalfree catalyst. RSC Adv 6: 72121-72126.

18. Prasad RN, Hawkins LR, Tietje K (1968) Potential antihypertensive agents.II. Unsymmetrically 1,4-disubstituted piperazines I. J Med Chem 11: 1144-1150. 
Citation: Chen T, Jiang H, Zhou J, Li Z, Huang W, et al. (2018) Synthesis of N-Substituted Benzamide Derivatives and their Evaluation as Antitumor Agents. Med Chem (Los Angeles) 8: 273-280. doi: 10.4172/2161-0444.1000524

19. Zhou JJ, Li YN, Sun HB, Tang ZK, Qi L, et al. (2017) Porous sillicaencapsulated and magnetically recoverable Rh NPs: a highly efficient, stable and green catalyst for the catalytic transfer hydrogenation with "slow-releasing" of stoichiometric hydrazine in water. Green Chem 19: 3400-3407.

20. Xie J, Yuan XG, Abdukader A, Zhu CJ, Ma J (2014) Visible-light-promoted radical C-H trifluoromethylation of free anilines. Org Lett 16: 1768-1771.

21. Lu HT, Geng ZY, Li JY, Zhou DP, Wu YS, et al. (2016) Metal-free reduction of aromatic nitro compounds to aromatic amines with $\mathrm{B}_{2}$ pin $_{2}$ in isopropanol. Org Lett 18: 2774-2776.

22. Shukla MK, Mahesh RK, Chaudhary C (1981) Synthesis of 1-benzoyl-4arylmethyl -piperazines as CNS Active Agents. Ind J Chem 20: 680-682.

23. Sun CL, Qu PP, Li F (2014) Rearrangement of aldoximes to amides in water under air atmosphere catalyzed by water-soluble iridium complex $\left[\mathrm{Cp}^{*} \operatorname{Ir}\left(\mathrm{H}_{2} \mathrm{O}\right)_{3}\right]$ [OTf $]_{2}$. Catal Sci Technol 4: 988-996.

24. Srinivasan S, Manisankar $P(2010)$ Formamide as an ammonia synthon in amination of acid chlorides. Syn Commun 40: 3538-3543.

25. Nisa M, Munawar MA, lqbal A, Ahmed A, Ashraf M, et al. (2017) Synthesis of novel 5-(aroylhydrazinocarbonyl)escitalopram as cholinesterase inhibitors. Eur J Med Chem 138: 396-406.

26. Marce P, Lynch J, Blacker AJ, Williams JMJ (2016) A mild hydration of nitriles catalysed by copper(II) acetate. Chem Comm 52: 1436-1438.

27. Altenkirk B, Israelstam SS (1962) Reaction of tert-butyl hypochlorite.IV. The reaction between tert-butyl hypochlorite and benzamides. Alten Israel 27: 4532-4534.

28. Taha M, Ismail NH, Imran S, Selvaraj M, Rahim A, et al. (2015) Synthesis of novel benzohydrazone-oxadiazole hybrids as $\beta$-glucuronidase inhibitors and molecular modeling studies. Bioorg Med Chem 23: 7394-404.

29. Yale HL, Losee K, Martins J, Holsing M, Perry FM, et al. (1953) Chemotherapy of experiment tuberculosis. VIII. The synthesis of acid hydrazides, their derivatives and related compounds. J Am Chem Soc 75: 1933-1942.

30. Zhang DT, Ma YT, Liu Y, Liu ZP (2014) Synthesis of sulfonylhydrazoneand acylhydrazone-substituted 8-ethoxy-3-nitro-2H-chromenes as potent antiproliferative and apoptosis inducing agents. Arch Pharm Chem 347: 576 588.

31. Upadhyaya K, Thakur RK, Shukla SK, Tripathi RP (2016) One-pot copper(I)catalyzed ligand/base-free tandem cyclooxidative synthesis of quinazolinones. J Org Chem 81: 5046-5055.

32. Kaniskan N, Kokten S, Celik I (2012) A new protocol for the synthesis of primary, secondary and tertiary anthranilamides utilizing $\mathrm{N}$-(2-aminoarylacyl) benzotriazoles. Arkivoc 8: 198-213.

33. Benthammadi S, Iraten S, Othman AA (2016) Synthetic studies and antibacterial activity of nucleobases and their $\mathrm{N}$ - and S-glucosides from 2-amino benzoic acid and its benzamido derivatives. Orient J Chem 32: 2567 2576.

34. Zhou YY, Wang BL, Di FJ, Xiong LX, Yang N, et al. (2014) Synthesis and biological activities of 2,3-dihydro-1,3,4-oxadiazole compounds and its derivatives as potential activator of ryanodine receptors. Bioorg Med Chem Lett 24: 2295-2299.

35. Wendt MD, Kunzer AR (2010) Ortho selectivity in $S_{A} A r$ substitutions of 2,4-dihaloaromatic compounds. Reactions with piperidine. Tetrahedral Lett 51: 641-644.

36. O'Brien AG, Horvath Z, Levesque F, Lee JW, Morgenstern AS, et al. (2012) Continuous synthesis and purification by direct coupling of flow reactor with simulated moving-bed chromatography. Angew Chemie 51: 7028-7030.

37. Orkenyi R, Eles J, Faigl F, Vincze P, Prechl A, et al. (2017). Continuous synthesis and purification by coupling a multistep flow reaction with centrifugal partition chromatography. Angew Chemie 56: 8742-8745. 